\title{
Erratum to: Safety and Tolerability of Levomilnacipran ER in Major Depressive Disorder: Results from an Open-Label, 48-Week Extension Study
}

\author{
Rajnish Mago • Giovanna Forero • William M. Greenberg • \\ Carl Gommoll $\cdot$ Changzheng Chen
}

Published online: 12 October 2013

(c) Springer International Publishing Switzerland 2013

Erratum to: Clin Drug Investig (2013) 33:761-771
DOI 10.1007/s40261-013-0126-5

Page 771, Reference 11, which previously read:

Bakish D, Gommoll C, Chen C, et al. Levomilnacipran ER $40 \mathrm{mg}$ and $80 \mathrm{mg}$ in major depressive disorder: a phase III, randomized, double-blind, fixed-dose, placebo-controlled study Int Clin Psychopharmacol. 2013 (in press).

Should read:

Bakish D, Bose A, Gommoll C, Chen C, Greenberg WM, et al. Levomilnacipran ER $40 \mathrm{mg}$ and $80 \mathrm{mg}$ in major depressive disorder: a phase III, randomized, double-blind, fixed-dose, placebo-controlled study. J Psychiatry Neurosci. 2013: doi: 10.11503/jpn.130040 (In Press)

The online version of the original article can be found under doi:10.1007/s40261-013-0126-5.

R. Mago $(\bowtie)$

Mood Disorders Program, Department of Psychiatry and Human

Behavior, Thomas Jefferson University, 833 Chestnut St., Suite

210 E, Philadelphia, PA 19107, USA

e-mail: rajnish.mago@jefferson.edu

G. Forero · W. M. Greenberg · C. Gommoll

Clinical Development, Forest Research Institute,

Jersey City, NJ, USA

C. Chen

Biostatistics, Forest Research Institute, Jersey City, NJ, USA 